\title{
De la difficulté de traduire Baudelaire en italien
}

\section{Mario Richter}

\section{(2) OpenEdition}

Journals

Édition électronique

URL : http://journals.openedition.org/rief/594

DOI : 10.4000/rief.594

ISSN : 2240-7456

\section{Éditeur}

Seminario di filologia francese

\section{Référence électronique}

Mario Richter, «De la difficulté de traduire Baudelaire en italien », Revue italienne d'études françaises [En ligne], 4 | 2014, mis en ligne le 15 décembre 2014, consulté le 23 avril 2019. URL : http:// journals.openedition.org/rief/594; DOI : 10.4000/rief.594

Ce document a été généré automatiquement le 23 avril 2019.

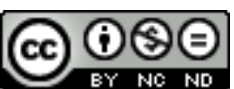

Les contenus de la RIEF sont mis à disposition selon les termes de la Licence Creative Commons Attribution - Pas d'Utilisation Commerciale - Pas de Modification 4.0 International. 


\title{
De la difficulté de traduire Baudelaire en italien
}

\author{
Mario Richter
}

1 Il n'est pas facile de trouver un poète français qui, plus que Baudelaire, ait suscité un aussi grand intérêt chez les traducteurs italiens. Ce sont également, pour la plupart, en particulier ces soixante dernières années, des poètes, et souvent des poètes remarquables (je pense à Diego Valeri et Alessandro Parronchi, mais surtout à Giorgio Caproni, Attilio Bertolucci, Carlo Muscetta, Gesualdo Bufalino, Cosimo Ortesta, Mario Luzi, Antonio Prete, Giovanni Raboni...). Si on voulait dresser ici une liste complète de toutes les traductions qui ont suivi la version en prose des Fleurs du mal, par ailleurs d'une assez bonne qualité, d'un écrivain peu connu, Riccardo Sonzogno, publiée en 1893 (et puis encore l'année suivante) $)^{1}$, cela exigerait sans doute plusieurs pages ${ }^{2}$.

2 Il faut plutôt se demander pour quelle raison la poésie de Baudelaire a obtenu un si grand succès en Italie.

3 Ce phénomène dépend, selon nous, de l'extraordinaire qualité poétique des Fleurs du mal, de l'influence profonde et du prestige que l'ouvrage a eus pour l'affirmation de l'esprit moderne. Il suffit de rappeler que des poètes et des écrivains tels que Rimbaud, Huysmans, Proust et Valéry (très appréciés en Italie) ont tous eu un véritable culte pour Baudelaire.

4 Toutefois il existe aussi, nous semble-t-il, une sorte d'attraction exercée par les Fleurs $d u$ mal due à ce qui pourrait être considéré comme une aisance ou une souplesse séduisante (nous ne disons pas facilité) qui invite à la traduction. Car les vers de Baudelaire présentent la plupart du temps une perfection unie, un rythme mesuré au goût essentiellement parnassien, un lexique transparent et courant même, parfois familier. Tout bien considéré (on l'a observé plusieurs fois), il n'y a pas de différence appréciable entre la langue poétique de Baudelaire et celle de Racine. Tout cela est tentant pour bien des lecteurs, et semble justement solliciter la traduction.

5 Mais, en réalité, tous ceux qui se laissent charmer par la poésie baudelairienne et s'essayent à la traduire se trouvent à devoir affronter un texte plein d'embûches. 
Nous allons en signaler ici quelques-unes parmi les plus significatives.

Commençons par la cinquième strophe du poème liminaire adressé Au Lecteur :

Ainsi qu'un débauché pauvre qui baise et mange

Le sein martyrisé d'une antique catin,

Nous volons au passage un plaisir clandestin

Que nous pressons bien fort comme une vieille orange ${ }^{3}$.

J'attire l'attention sur le verbe "mange ", mot que la fin du vers et la rime mettent décidément en relief. C'est un verbe que les traducteurs italiens se sont presque toujours efforcés de transformer en quelque chose d'autre, en une action qui leur a paru plus vraisemblable. Riccardo Sonzogno a commencé par croire convenable la traduction " morde » ("mord"). Il en a été de même pour De Nardis et Colesanti. Ortesta a cru qu'il vaut mieux traduire "succhia " ("suce"). Caproni a eu recours à un verbe de signification semblable, quoique plus rare : il a opté pour « smunge » ("épuiser", "saigner"). Muscetta a préféré choisir «biascica » (c'est-à-dire "mâchonne" ou "mordille"). Antonio Prete est allé plus loin : il a voulu exagérer en se décidant pour "divora » ("dévore"). Raboni a d'abord traduit « succhia e assapora » ("suce et savoure") (1973) pour se limiter plus tard à « assapora » (1996). En réalité, la traduction correcte, en l'occurrence, n'est que celle de Luciana Frezza, qui a opté pour une traduction littérale, c'est-à-dire «che bacia e che mangia ». En effet, le verbe manger ne signifie pas sucer, ni dévorer, ni mordre, ni savourer, etc., mais tout bonnement manger (en italien "mangiare"). Il est donc nécessaire que le traducteur respecte le choix de Baudelaire et, quand bien même cela pourrait lui paraître déplaisant, qu'il se résigne à accepter le banal et très matériel « mangia ».

Ce n'est en effet que par cette voie que l'on peut comprendre dans toute sa force la violence éminemment symbolique que Baudelaire veut ici imposer à sa pensée. Car il ne fait aucun doute que manger un sein nous éloigne de la réalité, de la normalité, de la vraisemblance ${ }^{4}$. Le sein d'une "antique catin », un sein qu'un "débauché pauvre »auquel le poète nous compare ${ }^{5}$ - «mange», renvoie de toute évidence à la réalité culturelle où l'on vit, réalité qui ne nous consent qu'un rapport clandestin et occasionnel avec des plaisirs avilissants, ceux que l'on peut justement tirer en mangeant le sein d'une antique prostituée comme s'il s'agissait d'une "vieille orange ». On remarquera que la rime mange: orange joue un rôle essentiel, qui assure la liaison entre les deux comparaisons de la strophe: la «vieille orange " nous renvoie à l'image du sein de l' " antique catin » et, en même temps, à la saveur "répugnante » que perçoit celui qui la "mange $»^{6}$. Ce n'est pas toutefois une orange que nous mangeons (ce qui serait plutôt normal, bien qu'il soit question d'une "vieille orange »), mais précisément - à travers la comparaison au "débauché pauvre" - un sein. Nous sommes là en présence d'un symbolisme à l'érotisme aberrant, répugnant, violent précisément, tout à fait insupportable, un érotisme délibérément dénué de toute vraisemblance, ce qui pourrait expliquer l'embarras qu'ont éprouvé les traducteurs italiens face au verbe "mange» ayant comme objet direct « le sein martyrisé d'une antique catin ».

En résumé, dans ce quatrain Baudelaire nous dit que notre réalité s'est horriblement détériorée, qu'elle est tombée tout entière dans le vice, dans la misère, dans la vieillesse, dans le vol, dans les plaisirs clandestins, fortuits (notez « au passage »), et paroxystiques. C'est ainsi que «nous » en arrivons à accomplir la plus concrète, la plus commune et élémentaire des actions, celle de manger un sein, précisément.

11 Un autre cas qui pose des problèmes de traduction est présent tout au début du 'sonnet' De profundis clamavi : 
J'implore ta pitié, Toi, l'unique que j'aime.

12 J'ai eu autrefois, au collège, un professeur qui aimait citer ce vers pour montrer qu'il est mauvais. Il indiquait le syntagme «l'unique que» pour dire : «Remarquez ce que-que: c'est intolérable ; un vrai poète ne devrait jamais faire de semblables fautes de goût ». Et il ajoutait l'axiome suivant : "Tout ce qui est poésie est chant » (par la suite, j'ai découvert qu'il s'agit là d'une affirmation du poète parnassien Théodore de Banville, l'un des amis de Baudelaire et l'auteur du Petit traité de poésie française).

13 Certes, de son point de vue, cet enseignant n'avait pas tort. Car ce que-que est effectivement une cacophonie. Et je me souviens qu'il considérait comme préférables d'autres tournures (par exemple : la seule que j'aime).

14 Or, relisant des années plus tard ce poème et le replaçant dans son contexte (c'est-à-dire dans Les Fleurs du mal de 1861), j'ai pu comprendre combien était insuffisante et même trompeuse l'esthétique d'euphonie avec laquelle cet enseignant jugeait la poésie et en particulier, pour le condamner, le vers de Baudelaire cité.

15 Je me suis rendu compte que l'adjectif par lui tant décrié (" unique») était, bien au contraire, pour le poète tout à fait nécessaire, étant donné que, dans ce cas-là, c'est le sens qui devait l'emporter sur tout autre aspect. Car ce à quoi Baudelaire tenait par-dessus tout, c'était une parfaite ambivalence entre le genre masculin et le féminin du «Toi » (objet de l'amour) auquel s'adresse celui qui implore la pitié. En effet, unique est en français (à la différence de l'italien unico, qui comporte le féminin unica) une épithète à double genre. J'ai remarqué que presque tous les traducteurs (sans doute encouragés par l'explication qu'a donnée dans son édition Antoine Adam, qui prétend que l'adjectif « unique » se réfère à Jeanne, la femme biographique qui, par ailleurs, dans les Fleurs du mal n'est jamais nommée sous ce nom) optent pour le genre féminin (ainsi, par exemple, De Nardis, Ortesta et Raboni et bien d'autres). Mais il s'agit là d'un choix arbitraire, d'une grave infidélité au texte (ils ont bien fait, en revanche, ceux qui - comme Bufalino, Frezza et Prete - ont traduit «Imploro la tua pietà, o Te, mio unico amore » et également Caproni, à qui on doit, malgré l'apparente incorrection « o Tu », cette solution : « Imploro la tua pietà, o Tu, unico essere che amo »).

16 Mais essayons de mieux comprendre le problème que le traducteur italien doit ici résoudre.

17 Considérons rapidement le texte qui se lit tout de suite avant (Une charogne). C'est un poème dans lequel le Poète ${ }^{7}$ invite une femme - une dame catholique de la bonne société qu'il vouvoie mais qu'il appelle son « âme » (« Rappelez-vous l'objet que nous vîmes, mon âme ») - à se rappeler qu'un été, au cours d'une promenade, leur arriva d'assister à la destruction que la " grande Nature » infligeait au cadavre d'un animal. Le Poète s'efforce de garder le souvenir de la forme de l'animal en putréfaction que les larves, les mouches et les vers étaient en train d'anéantir tout en donnant origine à un essaim bourdonnant d'autres vies. À la fin du poème, il s'adresse à la femme-âme pour lui dire que, quand elle aussi aura été enterrée après avoir reçu les «derniers sacrements » (confession, communion, extrême-onction), ce ne sera en fait que lui, le Poète, qui aura su garder « la forme et l'essence divine / De [s]es amours décomposés ». Il proclame ainsi, orgueilleusement, le pouvoir de la poésie et nie en même temps l'immortalité de l'âme. Il n'y a pas de doute que nous sommes là en présence d'un message qui n'a presque rien d'original : c'est celui qu'Horace, entre autres, a transmis à la littérature moderne, de Ronsard aux Parnassiens. Si Baudelaire avait conclu son livre par ces vers, sans doute son 
ouvrage n'aurait pour nous d'autre sens que celui des nombreux recueils d'habiles parnassiens de l'époque, s'en remettant à l'immortalité de la poésie et pour lesquels, justement, « tout ce qui est poésie est chant ».

Certes, on aurait eu un beau livre, mais d'une beauté dénuée de chaleur et de vie (une beauté qui, au fond, n'intéresse personne), une œuvre morte, belle comme peut être beau un « soleil de glace » (voir le vers $10 \mathrm{du}$ 'sonnet' De profundis clamavi).

$19 \mathrm{Au}$ contraire, après tant d'orgueil, heureusement le livre se poursuit de façon surprenante avec l'invocation à la pitié la plus désolée qui existe, précisément la prière judéo-chrétienne de la pénitence De profundis clamavi ad te, Domine (sixième psaume) unie à celle du Miserere (quatrième psaume).

Nous devons commencer par nous demander à qui maintenant le Poète s'adresse. Car ce vers ne nous permet pas de savoir avec précision (nous l'avons vu) si cet « unique » est masculin ou féminin. Qui peut donc être ce «Toi » (avec le T majuscule), cet Amour " unique »? Je crois qu'il n'y a d'autre réponse que celle-ci : étant donné le titre (qui renvoie au Dominus du psaume), «Toi » et donc « unique » doivent être considérés comme masculins, et ils se réfèrent à Dieu, le Dominus du psaume que, dans Une charogne, le Poète, qui a nié l'immortalité de l'âme, vient d'offenser. Mais, compte tenu du contexte (qui met en scène des femmes, dont la dernière est la "grande Nature", personnifiée par le $\mathrm{N}$ majuscule), «Toi » et donc « unique » doivent être considérés comme féminins, et ils semblent se référer, justement, à la " grande Nature ", que le Poète vient de contrarier au nom de la conservation individuelle à laquelle vise l'art.

On pénètre par là dans un espace typiquement baudelairien, un lieu où l'unité renvoie à la duplicité, un inconnu qui se dissimule dans les couples oppositifs du Dieu judéo-chrétien (masculin) et de la Nature (féminin), tous deux précédemment outragés par le Poète.

Il faut donc remarquer que Baudelaire a eu intentionnellement recours à l'ambivalence (en ce qui concerne le genre) de l'adjectif "unique», au risque de commettre, dans le contexte du vers, une cacophonie que justement bien des lecteurs ont dénoncée (« unique que ») et que, du reste, il lui aurait été bien facile d'éviter. Il s'agit d'une cacophonie ayant, elle aussi, sa raison d'être; car elle semble reproduire une sorte de sanglot, ce qui convient parfaitement à un homme en proie au repentir. Baudelaire montre ainsi de vouloir affirmer, dans ce cas-ci, la primauté du sens sur la perfection formelle chère à Gautier et aux Parnassiens. Ce qui revient à dire que le vers cacophonique s'avère strictement nécessaire au poème à condition qu'on le lise avec respect, c'est-à-dire en le considérant à la lueur de son contexte.

Examinons maintenant un autre cas d'ambivalence comportant une difficulté de traduction. C'est dans la seconde partie du «tableau parisien » La servante au grand cœeur ...:

Lorsque la bûche siffle et chante, si le soir,

Calme, dans le fauteuil je la voyais s'asseoir,

$\mathrm{Si}$, par une nuit bleue et froide de décembre,

Je la trouvais tapie en un coin de ma chambre,

Grave, et venant du fond de son lit éternel

Couver l'enfant grandi de son œil maternel,

Que pourrais-je répondre à cette âme pieuse,

Voyant tomber des pleurs de sa paupière creuse?

Dans la première partie de ce poème, le locuteur vient de nous dire que, s'il est vrai que les morts "dorment" et qu'ils restent donc dans un état de vie et de douleur, alors il 
devrait être vrai aussi qu'ils peuvent revenir parmi les vivants, sous la forme incorporelle des fantômes ou sous la forme corporelle des revenants. déterminante pour la réalisation de cette parfaite ambiguïté. En effet, si Baudelaire avait employé l'imparfait, il aurait été empêché de construire ensuite une proposition conditionnelle (qui se réalise dans le présent). Au contraire, en recourant à un présent de l'indicatif que rien n'interdit de percevoir comme un présent d'habitude (qui vaut pour toujours ${ }^{9}$ ), non seulement il a rendu possible l'ambiguïté, mais il a donné à la «bûche " qui « siffle et chante » dans la cheminée une valeur de continuité, de sécurité bourgeoise, dans le sens que cette "bûche » est encore là, maintenant, qui brûle comme autrefois pour réconforter les vivants. Avec ce présent, c'est comme s'il disait: "C'est toujours comme cela que cela se passe dans les familles aisées de Paris quand il fait froid». On comprend alors que cette «bûche» qui «siffle et chante» reflète le "mensonge" de l'organisation bourgeoise de la vie. Je veux dire qu'elle correspond à l'habitude précédente de porter des fleurs à ceux qui "dorment" dans le cimetière. Car le lecteur attentif des Fleurs du mal ne peut pas avoir oublié de quelle façon le Poète a réellement perçu, dans le Chant d'automne, le bruit que font les bûches quand, en automne, on les fait tomber sur le pavé des cours citadines pour assurer le chauffage de l'hiver ( J'entends déjà tomber avec des chocs funèbres / Les bois retentissant sur le pavé des cours [...] J'écoute en frémissant chaque bûche qui tombe $»)^{10}$. objectivement et pleinement active. À partir du vers qui suit («Grave, et venant du fond de son lit éternel »), la nature de la subordonnée commence à se déplacer - en raison du sens - de sa nature temporelle possible, pour assumer, presque uniquement, l'autre valeur, conditionnelle - rapportée au présent -, de la potentialité ou même de l'irréalité. J'ai dit «presque uniquement» parce que, si l'on examine de plus près, l'aspect évocateur (déterminé par le si de nature temporelle) ne semble pas disparaître tout à fait. Car, du 
point de vue enfantin évoqué par le Poète, la «servante au grand cœur» pouvait bien donner à l'enfant l'impression de venir d'un lit lointain et différent ( du fond de son lit»), d'une réalité à part, divinement protectrice, rassurante, comme si elle était précisément une divinité, capable, avec son "grand cœur", de protéger, de bercer, de consoler. Je crois que tout enfant peut se rappeler de lointaines illusions familiales de ce genre, quand la maman (ou celle qui la remplaçait) semblait une présence infaillible et éternelle, une parfaite présence divine ( de son lit éternel »).

On atteint là, sans aucun doute, un équilibre parfait entre le passé et le présent. En effet, seulement quand on a terminé la lecture de ce vers, on commence à comprendre avec suffisamment de clarté (vu aussi ce que l'on a appris dans la première partie du poème) de quel lieu pourrait, par hypothèse, venir la " servante ", je veux dire venir en ce moment-ci, c'est-à-dire dans le présent de l'énonciation (le sens hypothétique imminent, et non plus temporel, du si jouant alors avec plus de force) : la « servante » pourrait venir maintenant « du fond de son lit », et on devra comprendre non plus du lit «éternel » (au sens de divin) qu'autrefois la "servante » réchauffait aussi dans la tranquille petite maison bourgeoise et qui, actuellement, est réchauffé par les vivants, mais bien du lit euphémique et généralement chrétien où la domestique est en train de "dormir", "sous une humble pelouse », son sommeil éternel, sa requiem ceternam. Le sens hypothétique, déterminé maintenant par la principale au conditionnel présent («Que pourrais-je...»), nous fait passer d'une précédente dimension naturelle possible (le souvenir de la domestique quand elle était vivante, aspect de si temporel) à une dimension surnaturelle (rendue légitime par la survie chrétienne), c'est-à-dire à un retour actuel de la « servante » défunte (ou plutôt, "dormeuse"!).

Bien habile le traducteur italien qui saura rendre dans sa langue cette ambivalence, à mon avis - comme j'espère l'avoir suffisamment montré - très suggestive en raison de son sens riche, si éminemment poétique! Jusqu'à présent, personne, à ma connaissance, n'a su traduire ce double sens de la phrase, se limitant à son seul aspect hypothétique ${ }^{11}$.

31 Il y a un autre endroit où le traducteur italien doit faire face à une difficulté apparemment insurmontable. C'est dans les deux vers sur lesquels s'achève Le Crépuscule du matin :

Et le sombre Paris, en se frottant les yeux,

Empoignait ses outils, vieillard laborieux.

Pour les traduire de façon correcte, il faut d'abord avoir compris la raison profonde qui gouverne ce poème.

On sait qu'il y a un thème tout à fait central dans Les Fleurs du mal : c'est la profonde et grave division (incompréhension) entre l'homme et la femme. Ce thème devient particulièrement chargé de sens dans les "Tableaux parisiens", c'est-à-dire dans la partie de l'ouvrage où le lecteur se trouve devant Paris, la ville qui, sans doute plus que toute autre au XIX ${ }^{e}$ siècle, exprime le progrès en tant que conquête masculine (une culture intellectualiste fondée sur l'écriture et sur la conservation).

Le Crépuscule du matin, qui conclut cette section, a l'un de ses points cruciaux précisément dans cette division. Il suffit de considérer le vers que voici, par lequel s'achève la deuxième des quatre parties dont se compose le poème :

Et l'homme est las d'écrire et la femme d'aimer.

Ce vers nous fait savoir qu'au point du jour, à Paris, l'homme et la femme sont tous deux fatigués et dégoûtés («las ») de leurs activités nocturnes respectives, l'écriture et l'amour, 
qui les divisent. On remarquera que la forme métrique respecte également cette union séparée. Car ce dernier vers de la deuxième partie d'un poème bâti sur une succession de distiques à rimes plates reste interrompu à sa première rime, et ne se termine pas dans la forme achevée du distique. Tout comme l'homme et la femme, le couple de rimes est séparé, divisé. Plus précisément, il s'unit, si l'on peut dire, dans la séparation (dans la douleur), puisque la rime du distique ne se complète qu'avec le premier vers de la partie suivante.

Ce qui est curieux, c'est que cette division anomale du distique ne semble pas avoir retenu l'attention des commentateurs. Car, devant cette infraction à la norme (qui attire l'attention), il est nécessaire de se demander : que signifie donc exactement l'information singulière et inattendue de ce vers qui, à cause du système des rimes (le distique), contient le sens d'une union désunie?

Précisons qu'il s'agit d'une situation générale: Baudelaire ne dit pas, par exemple, «l' écrivain est las d'écrire et sa femme d'aimer ", ou quelque chose du même genre. Dans ce cas, tout rentrerait tranquillement dans la norme d'un couple en dehors de la norme (c'est-à-dire un écrivain qui néglige sa femme ou sa compagne parce qu'il est entièrement absorbé, même la nuit - grâce à la lumière artificielle de la lampe -, par son activité). Baudelaire dit ici : « l'homme [...] et la femme », c'est-à-dire qu'il présente comme général et normal ce rapport de couple qui est considéré comme exceptionnel. C'est donc, approximativement, comme s'il disait : "À Paris, tous les hommes se comportent la nuit comme un écrivain : ils pensent à leur activité et ils négligent leur femme qui a besoin d'amour ».

Pour autant que cette information puisse paraitre étrange, il faut donc l'accepter pour ce qu'elle est: tous les parisiens (de sexe masculin) écrivent la nuit («Et l'homme est las d'écrire »). Ce sont donc tous des fous, des types étranges, exactement comme en général les gens du peuple jugent fou, ou du moins excentrique, l'écrivain qui écrit la nuit et néglige sa femme ou sa compagne qui a besoin d'amour. Qu'est-ce que cela signifie ? Cela signifie que même celui qui n'a pas l'impression de se comporter comme un fou ou comme un excentrique (comme un écrivain, ou comme un poète), en fait participe lui aussi à une réalité culturelle née de l'activité littéraire, de l'écriture, de l'encre. Ceci étant, les femmes aiment beaucoup, jusqu'à en être « lasses ». Un poème précédent, Les petites vieilles, nous a fait savoir que les femmes, au cours de leur vie, sont unies par un besoin d'amour qui n'a pu s'exprimer que dans un absurde "dévouement » pour les hommes (maris et enfants), au point que leurs yeux sont des puits «faits d'un million de larmes ».

Venons-en maintenant à la conclusion du poème.

Il a été nécessaire d'insister sur la division entre l'homme et la femme pour dire combien il est fondamental, pour bien traduire ce poème, de conserver la distinction entre ce qui est masculin et ce qui est féminin. Car c'est précisément sur cette distinction que Baudelaire a voulu bâtir son texte et faire passer la spécificité de son message.

Or, le traducteur italien (à condition, bien entendu, qu'il ait compris la raison profonde de ce poème) se trouve devant un problème dont la solution s'avère particulièrement difficile et peut-être même impossible.

Paris - qui en italien (Parigi) est du genre féminin - est en français un nom masculin. Personne ne peut lui attribuer un autre genre. Pour Baudelaire, poète français, ceci est un fait de toute première importance. En effet Paris est comparé à un «vieillard», dont 
l'idole la plus respectée est le travail (parce qu'il adore le « dieu de l'Utile, implacable et serein", cf. J'aime le souvenir de ces époques nues): c'est justement un "vieillard laborieux ». L'aurore, au contraire, bien qu'elle soit si ancienne, c'est une fille qui voudrait se reproposer chaque jour avec sa délicatesse, avec sa merveille de jeune femme. Mais Paris, le « vieillard laborieux » (ainsi que l'homme qui écrit) la désavoue.

Il est donc indispensable, pour le traducteur italien, que le nom propre dont il dispose dans sa langue (Parigi) soit de genre masculin, autrement dit il faut qu'il soit un « vieillard » entièrement consacré au travail.

Les meilleurs traducteurs italiens se sont, bien sûr, efforcés de respecter cette spécificité du texte. Mais je pense qu'ils n'ont pas réussi à bien surmonter cet obstacle. Car, en italien, on ne pourra jamais dire que Parigi (nom féminin) est « fosco » (« il fosco Parigi, vecchio laborioso ", traduit Sonzogno, qui a certes bien compris la nécessité de garder le masculin du texte original), ou traduire que Parigi est un « cupo vegliardo » (Ortesta), ou encore un «laborioso vecchio " (De Nardis). Dans ces traductions, ou bien l'on fait une entorse à la langue italienne (comme c'est le cas de Sonzogno, qui transforme le nom féminin Parigi en le faisant masculin), ou bien on crée un monstre à double genre (Parigivecchio), alors que, dans son poème, Baudelaire a veillé à garder le masculin et le féminin bien séparés et en opposition.

Ces quelques exemples devraient suffire à nous faire comprendre combien la traduction des Fleurs du mal, malgré la facilité apparente de cet ouvrage, est en fait une entreprise pleine de pièges. Baudelaire exige toujours de son traducteur la plus grande attention et le plus grand respect ${ }^{12}$, mais surtout il lui demande la plus grande humilité, la seule qui lui rende possible l'accès effectif aux terres inconnues que ce poète, de façon géniale et subtile, ne cesse d'explorer et de nous faire connaître.

\section{NOTES}

1. Riccardo Sonzogno est le petit-fils d'Edoardo Sonzogno, fondateur de la célèbre maison d'édition milanaise.

2. Il suffit de voir les listes, assez exhaustives, dans la Bibliographie du « Meridiano » procuré par Giovanni Raboni (Mondadori, 1996), ou la bibliographie jointe à la traduction de Cosimo Ortesta (Giunti, 1996).

3. Toutes les citations sont tirées de Baudelaire, CEuvres complètes, texte établi, présenté et annoté par Claude Pichois, Paris, Gallimard, « Bibliothèque de la Pléiade », 1975.

4. Il est vrai que l'expression figurée "manger de baisers" existe, mais je ne crois pas que Baudelaire veuille ici répéter la même action que celle qu'il vient d'exprimer avec le verbe baiser.

5. On considère le singulier débauché comme un collectif.

6. Plus haut on lit le vers : «Aux objets répugnants nous trouvons des appâts ».

7. Il va sans dire que par Poète j'entends le personnage principal de l'ouvrage.

8. "Si peut marquer un rapport de temps et signifier 'toutes les fois que' : Si je dis oui, elle dit non. S'il pleuvait, nous ne sortions pas " (Grevisse, Le Bon usage, 984, e).

9. Cf. Grevisse, Le Bon usage, $715,1^{\circ}$ et 2. 
10. Pour la lecture de Chant d'automne, je me permets de renvoyer à mon ouvrage Baudelaire. Les Fleurs du Mal. Lecture intégrale, Genève, Slatkine, 2001, p. 533 sq.

11. Voici, par exemple, la traduction de l'un des plus sensibles lecteurs italiens de Baudelaire, Giorgio Caproni : «Se io la vedessi sedere nella sua poltrona, calma, quando, la sera, sibila e canta il cocco ; se, in una notte turchina e fredda di dicembre, me la trovassi in camera, rannicchiata in un angolo, grave, uscita dal fondo del suo letto eterno a covare con materni occhi il bimbo cresciuto, che cosa potrei rispondere a quell'anima pia, vedendole sgorgare le lacrime dalle vuote palpebre? " (Charles Baudelaire, I fiori del male, traduzione di Giorgio Caproni, introduzione e commento di Luca Pietromarchi, con testo a fronte, testo della traduzione stabilito da Luciano Carcereri, Venezia, Marsilio, 2008, p. 269).

12. Par exemple, traduisant l'Hymne à la Beauté, Giovanni Raboni a cru bon de changer en indirect le discours direct avec lequel Baudelaire a voulu donner la parole à l'éphémère ("L'éphémère ébloui vole vers toi, chandelle, / Crépite, flambe et dit: Bénissons ce flambeau !», v. 17-18). Si l'on considère que, parmi la quantité de personnifications prestigieuses et tragiques qui sont mobilisées dans l' Hymne (Destin, Horreur, Meurtre, Satan, Dieu, Ange, Sirène, Beauté), le seul "personnage" qui ait le privilège de parler est justement l'éphémère (et, qui plus est, cet insecte modeste parle aussi au nom des autres, assumant presque la fonction de guide, de célébrant, comme le prêtre dans la liturgie catholique), on se rend bien compte de la valeur irremplaçable qu'a ici le discours direct, et combien sa suppression est regrettable.

\section{INDEX}

Mots-clés : Baudelaire (Charles), traduction, Fleurs du mal, Au Lecteur, De profundis clamavi, Servante au grand cœur, Crépuscule du matin 